\title{
Bayesian Inference for Step-Stress Partially Accelerated Competing Failure Model under Type II Progressive Censoring
}

\author{
Xiaolin Shi, ${ }^{1}$ Fen Liu, ${ }^{2}$ and Yimin Shi ${ }^{2}$ \\ ${ }^{1}$ School of Electronic Engineering, Xian University of Posts and Telecommunications, Xian 710121, China \\ ${ }^{2}$ Department of Applied Mathematics, Northwestern Polytechnical University, Xi'an 710072, China \\ Correspondence should be addressed to Xiaolin Shi; linda20016@163.com
}

Received 4 July 2016; Revised 4 November 2016; Accepted 17 November 2016

Academic Editor: José A. Sanz-Herrera

Copyright (C) 2016 Xiaolin Shi et al. This is an open access article distributed under the Creative Commons Attribution License, which permits unrestricted use, distribution, and reproduction in any medium, provided the original work is properly cited.

\begin{abstract}
This paper deals with the Bayesian inference on step-stress partially accelerated life tests using Type II progressive censored data in the presence of competing failure causes. Suppose that the occurrence time of the failure cause follows Pareto distribution under use stress levels. Based on the tampered failure rate model, the objective Bayesian estimates, Bayesian estimates, and EBayesian estimates of the unknown parameters and acceleration factor are obtained under the squared loss function. To evaluate the performance of the obtained estimates, the average relative errors (AREs) and mean squared errors (MSEs) are calculated. In addition, the comparisons of the three estimates of unknown parameters and acceleration factor for different sample sizes and different progressive censoring schemes are conducted through Monte Carlo simulations.
\end{abstract}

\section{Introduction}

The competing failure model is commonly used in medical studies or reliability analysis. Due to the complexity in the internal structure and the external working environment, there are often many causes of the product failure. Each of these causes is possible to result in final failure of the product. This is called competing failure. In recent years, some of authors have investigated the competing failure models. Han and Kundu [1] studied the statistical analysis of the stepstress accelerated life test (SSALT) with competing risks for failure from the generalized exponential distribution under Type I censoring, and the point estimation and confidence interval of the distribution parameters are obtained. In the case that failure follows the lognormal distribution, Roy and Mukhopadhyay [2] investigated the maximum likelihood analysis of the accelerated life test of competing failure products using the EM algorithm. Zhang et al. [3] applied the copula model to combine the marginal distribution of the competing failure models with the time to failure distribution of the products and derived a general statistical model for accelerated life test (ALT) with s-dependent competing failures. Shi et al. [4] considered a constant-stress accelerated life test (CSALT) with competing risks for failure from exponential distribution under progressive Type II hybrid censoring. The maximum likelihood estimator and Bayes estimator of the parameter were derived. $\mathrm{Xu}$ and Tang [5] and $\mathrm{Wu}$ et al. [6] considered different inferential issues regarding the constant-stress accelerated competing failure models when the lifetime of different risk factors follows Weibull distribution. Other research results can be found in $[7,8]$.

The above references considered competing failure models in the case of CSALT or SSALT. Based on the CSALT or SSALT to analyze the life characteristics of product, we need to use the relationship between product life and stress levels, that is, acceleration model. But, in engineering practice, the accelerated model is not always known, such as the newly developed products. In order to solve this problem, the partially accelerated life test (PALT) is more suitable to be performed. There are two types of PALT, namely, constantstress PALT (CSPALT) and step-stress PALT (SSPALT). The latter allows for changing the stress from normal level to accelerated level at a specific time point, which contributes to getting the information of the products' lifetimes quickly. Under the PALT, Abd-Elfattah et al. [9] and Ismail [10, 11] 
derived the maximum likelihood estimates and Bayesian estimates of the acceleration factor and unknown parameters for different lifetime distributions. Abushal and Soliman [12] studied the MLE and Bayesian estimate of the parameters for the Pareto distribution of the second type under progressive censoring data for constant PALT. Ismail and Sarhan [13] discussed optimal design of step-stress life test with progressively Type II censored exponential data. But few authors have studied competing failure models from this perspective and especially from the Bayesian viewpoint.

The Bayesian method has certain accurate advantages when sample size is small. When little knowledge about the prior information is obtained, we can derive the objective Bayesian estimates based on noninformative priors, such as the Jeffreys prior and reference prior. For more details, see Jeffreys [14]. Guan et al. [15] derived the objective Bayesian estimates of unknown parameters for bivariate MarshallOlkin exponential distribution, respectively. Under Type I censoring, $\mathrm{Xu}$ and Tang [5] considered the objective Bayesian inference for the accelerated competing failure models. Instead, with historical data or experience of some experts available, the subjective Bayesian approach on the basis of informative prior distributions can be implemented, while if the hyperparameters of the informative prior distributions are unknown, we are able to use E-Bayesian method proposed by Han $[16,17]$. By assuming the hyperparameters follow certain prior distributions, the E-Bayesian estimate can be obtained as the expectation of the Bayesian estimate of the unknown parameter.

In life testing and reliability studies, the experimenter may not always obtain complete information on failure times for all experimental units. Thus, censoring is common to be performed. A censoring scheme (CS), which can balance between total time spent for the experiment, number of units used in the experiment, and the efficiency of statistical inference based on the results of the experiment, is desirable [12]. Compared to the conventional Type I and Type II censoring scheme, Type II progressive censoring scheme provides higher flexibility to the experimenter by allowing for product to be removed at nonterminal time points. This allowance may be desirable when some degradation-related information from live test specimens needs to be collected and analyzed or when scarce testing facilities need to be released for other experimentation. In recent years, some of researchers engaged in reliability research using progressively censored data, such as Abushal and Soliman [12] and Ismail and Sarhan [13].

The Pareto distribution has found widespread use as a model for various socioeconomic phenomena; see [18, 19]. The Pareto distribution has also been used in reliability and lifetime modelling (see [20-22]). In this paper, we will consider Bayesian analysis for the step-stress partially accelerated competing failure model from Pareto distribution under Type II progressive censoring. The rest of this paper is organized as follows. In Section 2, under Type II progressive censoring scheme, a step-stress partially accelerated competing failure model from Pareto distribution is described and some basic assumptions are given. In Section 3, we obtain the objective Bayesian estimates of the acceleration factor and unknown parameters based on the noninformative prior distribution. Furthermore, the Bayesian and corresponding E-Bayesian estimates are provided. In Section 4, we provide two real data sets to justify the use of the Pareto distribution in engineering practice. The simulation results of all proposed methods for different sample sizes and for different progressive censoring schemes are presented in Section 5, and some conclusions are presented in Section 6.

\section{Assumptions and Model Description}

Throughout the paper, we make the following basic assumptions:

(1) The failure of a product occurs only due to one of the 2 independent competing failure causes with lifetimes $T_{1}$ and $T_{1}$. Then the lifetime of the product is $T=$ $\min \left(T_{1}, T_{2}\right)$.

(2) Two stress levels $S_{0}$ and $S_{1}$ are used in SSPALT $\left(S_{0}<\right.$ $S_{1}$ ), where $S_{0}$ is use stress level and $S_{1}$ is accelerated stress level.

(3) Let $T_{1 j}$ be the occurrence time of the $j$ th failure cause under stress levels $S_{0}$ and $T_{2 j}$ be the occurrence time of the $j$ th failure cause under stress levels $S_{1}, j=$ 1,2. The times $T_{1 j}$ and $T_{2 j}$ are independent. Also, $T_{1 j}$ follows a Pareto distribution with the following hazard rate function (HRF) and reliability function:

$$
\begin{aligned}
& r_{1 j}(t)=\frac{\alpha_{j}}{t}, \quad t \geq \theta_{j} \\
& S_{1 j}(t)=\theta_{j}^{\alpha_{j}} t^{-\alpha_{j}}, \quad t \geq \theta_{j}, \quad j=1,2,
\end{aligned}
$$

where $\theta_{j}>0$ is the scale parameter and $\alpha_{j}>0$ is the shape parameter, $j=1,2$.

(4) Tampered failure rate model holds: that is, the HRF $r_{1 j}$ is proportional to $r_{2 j}$. Thus, the HRF of the $j$ th failure cause in SSPALT can be expressed as

$$
r_{j}^{*}(t)= \begin{cases}r_{1 j}(t)=\frac{\alpha_{j}}{t}, & \theta_{j} \leq t \leq \tau, \\ r_{2 j}(t)=\frac{\alpha_{j} \lambda}{t}, & t>\tau, \lambda>1,\end{cases}
$$

where $\tau$ is prefixed number, $\tau \in(0, \infty)$, and $\lambda$ is acceleration factor. The corresponding reliability function is

$$
S_{j}^{*}(t)= \begin{cases}S_{1 j}(t)=\theta_{j}^{\alpha_{j}} t^{-\alpha_{j}}, & \theta_{j} \leq t \leq \tau, \\ S_{2 j}(t)=\theta_{j}^{\alpha_{j}} \tau^{\alpha_{j}(\lambda-1)} t^{-\alpha_{j} \lambda}, & t>\tau, \lambda>1 .\end{cases}
$$

Under Type II progressive censored scheme, SSPALT is described as follows: $n$ identical products are placed on the test under the stress level $S_{0}$. At the time of the first failure, $R_{1}$ products are randomly removed from the remaining $n-1$ products, and the failure cause index $c_{1}$ is recorded. Similarly, at the time of the second failure, $R_{2}$ products are randomly 
removed from the remaining $n-2-R_{1}$ products, and the failure cause index $c_{2}$ is recorded. The test continues until time point $\tau$, the stress levels are changed from $S_{0}$ to $S_{1}$. Under the stress level $S_{1}$, the remaining $n-N_{1}-R_{1}-R_{2}-\cdots-R_{N 1}$ products continue to be tested. Suppose that the total number of failures under the stress level $S_{0}$ is $N_{1}$. At the time of the $\left(N_{1}+1\right)$ th failure, $R_{N 1+1}$ products are randomly removed from the remaining $n-\left(N_{1}+1\right)-R_{1}-R_{2}-\cdots-R_{N 1}$ products, and the failure cause index $c_{N_{1}+1}$ is recorded. The test continues until the $m$ th failure is observed and the failure cause index $c_{m}$ is recorded. At this time, all the remaining $R_{m}=n-m-R_{1}-R_{2}-\cdots-R_{m-1}$ products are removed and the test terminates, where $m, R_{i}(i=1,2, \ldots, m-1)$ are prefixed number $\left(N_{1}<m<n\right)$ and $c_{i} \in\{1,2\}, i=1,2, \ldots, m$. We denote the time of the $i$ th failure by $t_{i}$ and assume that each failure time along with the failure cause index is observed, and there is at least one failure caused by the $j$ th $(j=1,2)$ failure cause under stress level $S_{k}(k=0,1)$. Therefore, observed data can be denoted as $\left(t_{1}, c_{1}\right),\left(t_{2}, c_{2}\right), \ldots,\left(t_{m}, c_{m}\right)$. Then the likelihood function of the failure samples caused by the $j$ th failure cause is

$$
\begin{aligned}
& L_{j} \\
& \qquad \propto \prod_{i=1}^{N_{1}} r_{1 j} \delta_{j}\left(c_{i}\right)\left(t_{i}\right) S_{1 j}^{1+R_{i}}\left(t_{i}\right) \prod_{i=N_{1}+1}^{m} r_{2 j} \delta_{j}\left(c_{i}\right)\left(t_{i}\right) S_{2 j}^{1+R_{i}}\left(t_{i}\right) \\
& \quad=\alpha_{j}^{n_{j}} \theta_{j}^{\alpha_{j} n} \lambda^{n_{2 j}} U_{1 j} U_{2 j} W_{1}^{-\alpha_{j} \lambda} W_{2}^{\alpha_{j}(\lambda-1)}
\end{aligned}
$$

where

$$
\begin{aligned}
\delta_{j}\left(c_{i}\right) & = \begin{cases}1, & c_{i}=j, \\
0, & c_{i} \neq j,\end{cases} \\
U_{1 j} & =\prod_{i=1}^{N_{1}} t_{i}^{-\delta_{j}\left(c_{i}\right)-\alpha_{j}\left(1+R_{i}\right)}, \\
U_{2 j} & =\prod_{i=N_{1}+1}^{m} t_{i}^{-\delta_{j}\left(c_{i}\right)} \\
W_{1} & =\prod_{i=N_{1}+1}^{m} t_{i}^{\left(1+R_{i}\right)}, \\
W_{2} & =\tau^{A(m)}, \\
n_{j} & =n_{1 j}+n_{2 j}, \\
n_{1 j} & =\sum_{i=1}^{N_{1}} \delta_{j}\left(c_{i}\right) \\
n_{2 j} & =\sum_{i=N_{1}+1}^{m} \delta_{j}\left(c_{i}\right) \\
m) & =\sum_{i=N_{1}+1}^{m}\left(1+R_{i}\right)
\end{aligned}
$$

Based on assumptions (1) and (4), we derive the fulllikelihood function as

$$
\begin{gathered}
L\left(t \mid \lambda, \theta_{1}, \theta_{2}\right)=\prod_{j=1}^{2} L_{j} \propto\left(\prod_{j=1}^{2} \alpha_{j}^{n_{j}} U_{1 j} U_{2 j} \theta_{j}^{\alpha_{j} n}\right) \\
\cdot \lambda^{m-N_{1}} W_{1}^{-\left(\alpha_{1}+\alpha_{2}\right) \lambda} W_{2}^{\left(\alpha_{1}+\alpha_{2}\right)(\lambda-1)} .
\end{gathered}
$$

\section{Bayesian Analysis}

In this section, the Bayesian method is employed to estimate the scale parameters and acceleration factor when the shape parameters are known. Depending on whether the prior information is available or not, we obtain the objective Bayesian estimates, Bayesian estimates, and E-Bayesian estimates of the unknown parameters and acceleration factor under the squared loss function, respectively.

3.1. Objective Bayesian Estimates. When little knowledge about the prior information is obtained, the objective Bayesian approach is an alternative way. We assume that the joint prior distribution of the parameters $\left(\lambda, \theta_{1}, \theta_{2}\right)$ is

$$
\pi_{o}\left(\lambda, \theta_{1}, \theta_{2}\right) \propto \frac{1}{\lambda \theta_{1} \theta_{2}} .
$$

From (6) and (7), the posterior density functions of the parameters $\lambda, \theta_{1}$, and $\theta_{2}$ can be given by $\pi_{o}\left(\lambda, \theta_{1}, \theta_{2} \mid t\right) \propto$ $L\left(t \mid \lambda, \theta_{1}, \theta_{2}\right) \pi_{o}\left(\lambda, \theta_{1}, \theta_{2}\right)$. Then the marginal posterior density functions of the parameters $\lambda, \theta_{1}$, and $\theta_{2}$ can be obtained, respectively:

$$
\begin{aligned}
& \pi_{o}(\lambda \mid t)=D_{1} \lambda^{m-N_{1}-1} W_{1}^{-\left(\alpha_{1}+\alpha_{2}\right) \lambda} W_{2}^{\left(\alpha_{1}+\alpha_{2}\right) \lambda}, \quad \lambda>1, \\
& \pi_{o}\left(\theta_{1} \mid t\right)=D_{2} \theta_{1}^{\alpha_{1} n-1}, \quad 0<\theta_{1}<t_{11}, \\
& \pi_{o}\left(\theta_{2} \mid t\right)=D_{3} \theta_{2}^{\alpha_{2} n-1}, \quad 0<\theta_{2}<t_{21},
\end{aligned}
$$

where $t_{k 1}$ is the first-failure time caused by failure cause $k(k=1,2)$ and $D_{1}=\left[\int_{1}^{\infty} \lambda^{m-N_{1}-1} W_{1}^{-\left(\alpha_{1}+\alpha_{2}\right) \lambda} W_{2}^{\left(\alpha_{1}+\alpha_{2}\right) \lambda} d \lambda\right]^{-1}$, $D_{2}=\left[\int_{0}^{t_{11}} \theta_{1}^{\alpha_{1} n-1} d \theta_{1}\right]^{-1}$, and $D_{3}=\left[\int_{0}^{t_{21}} \theta_{2}^{\alpha_{1} n-1} d \theta_{2}\right]^{-1}$.

Under the squared loss function, the objective Bayesian estimates of the parameters $\lambda, \theta_{1}$, and $\theta_{2}$ can be obtained as

$$
\begin{aligned}
& \hat{\lambda}_{\mathrm{OBS}}=D_{1} \int_{1}^{\infty} \lambda^{m-N_{1}} W_{1}^{-\left(\alpha_{1}+\alpha_{2}\right) \lambda} W_{2}^{\left(\alpha_{1}+\alpha_{2}\right) \lambda} d \lambda, \\
& \widehat{\theta}_{j \mathrm{OBS}}=\alpha_{j} n t_{j 1}\left(\alpha_{j} n+1\right)^{-1}, \quad j=1,2 .
\end{aligned}
$$

It is obvious to see that (9) does not have explicit form. So we obtain the Bayesian estimate of $\lambda$ by numerical method. Let $f(\lambda)=\lambda^{m-N_{1}-1} W_{1}^{-\left(\alpha_{1}+\alpha_{2}\right) \lambda} W_{2}^{\left(\alpha_{1}+\alpha_{2}\right) \lambda}, V=\lambda\left(\alpha_{1}+\right.$ $\left.\alpha_{2}\right) \log \left(W_{1} / W_{2}\right)$, and then

$$
\begin{gathered}
\int_{1}^{\infty} \lambda^{m-N_{1}-1} W_{1}^{-\left(\alpha_{1}+\alpha_{2}\right) \lambda} W_{2}^{\left(\alpha_{1}+\alpha_{2}\right) \lambda} d \lambda=\int_{0}^{\infty} f(\lambda) d \lambda \\
-\int_{0}^{1} f(\lambda) d \lambda=\left[\left(\alpha_{1}+\alpha_{2}\right) \log \left(\frac{W_{1}}{W_{2}}\right)\right]^{-\left(m-N_{1}\right)}
\end{gathered}
$$




$$
\begin{aligned}
& \cdot\left[\int_{0}^{\infty} V^{m-N_{1}-1} e^{-V} d V-\int_{0}^{V^{*}} V^{m-N_{1}-1} e^{-V} d V\right] \\
& =\left[\left(\alpha_{1}+\alpha_{2}\right) \log \left(\frac{W_{1}}{W_{2}}\right)\right]^{-\left(m-N_{1}\right)} \\
& \cdot\left[\Gamma\left(m-N_{1}\right)-\int_{0}^{V^{*}} V^{m-N_{1}-1} e^{-V} d V\right],
\end{aligned}
$$

where $V^{*}=\left(\alpha_{1}+\alpha_{2}\right) \log \left(W_{1} / W_{2}\right)$. Similarly we get

$$
\begin{gathered}
\int_{1}^{\infty} \lambda f(\lambda) d \lambda=\int_{0}^{\infty} \lambda f(\lambda) d \lambda-\int_{0}^{1} \lambda f(\lambda) d \lambda \\
=\left[\left(\alpha_{1}+\alpha_{2}\right) \log \left(\frac{W_{1}}{W_{2}}\right)\right]^{-\left(m-N_{1}+1\right)} \\
\cdot\left[\Gamma\left(m-N_{1}+1\right)-\int_{0}^{V^{*}} V^{m-N_{1}} e^{-V} d V\right] .
\end{gathered}
$$

Thus, under the squared loss function, the objective Bayesian estimates of the parameters $\lambda$ can be obtained as

$$
\begin{aligned}
\hat{\lambda}_{\mathrm{OBS}} & =D_{1} \int_{1}^{\infty} \lambda^{m-N_{1}} W_{1}^{-\left(\alpha_{1}+\alpha_{2}\right) \lambda} W_{2}^{\left(\alpha_{1}+\alpha_{2}\right) \lambda} d \lambda=\frac{\int_{1}^{\infty} \lambda f(\lambda) d \lambda}{\int_{1}^{\infty} f(\lambda) d \lambda} \\
& =\frac{\left[\left(\alpha_{1}+\alpha_{2}\right) \log \left(W_{1} / W_{2}\right)\right]\left[\Gamma\left(m-N_{1}+1\right)-\int_{0}^{V^{*}} V^{m-N_{1}} e^{-V} d V\right]}{\left[\Gamma\left(m-N_{1}\right)-\int_{0}^{V^{*}} V^{m-N_{1}-1} e^{-V} d V\right]} .
\end{aligned}
$$

About the integrals $\int_{0}^{V^{*}} V^{m-N_{1}} e^{-V} d V$ and $\int_{0}^{V^{*}} V^{m-N_{1}-1} e^{-V} d V$, the compound trapezoidal integral formula can be used to obtain integral values. The specific steps are as follows: the interval $\left[0, V^{*}\right]$ is divided into $n$ equal subintervals and each length of subinterval is $h=V^{*} / n$. Then, from the compound trapezoidal integral formula, we get

$$
\begin{aligned}
& \int_{0}^{V^{*}} g(V) d V \\
& \quad \frac{h}{2}\left[g(0)+2 \sum_{k=1}^{n-1} g\left(\frac{V^{*} k}{n}\right)+g\left(V^{*}\right)\right], \\
& \int_{0}^{V^{*}} f(V) d V \\
& \quad \frac{h}{2}\left[f(0)+2 \sum_{k=1}^{n-1} f\left(\frac{V^{*} k}{n}\right)+f\left(V^{*}\right)\right],
\end{aligned}
$$

where $g(V)=V^{m-N_{1}} e^{-V}$ and $f(V)=V^{m-N_{1}-1} e^{-V}$. The above two integration results are substituted into (12), and the objective Bayesian estimates of the parameters $\lambda$ can be obtained.

3.2. Bayesian Estimates. With historical data or experts' experience available, the informative prior could be a better option. Following Doostparast et al. [23], a convenient prior family of distributions for $\theta_{j}(j=1,2)$ is provided by the power function distributions with probability density function

$$
\pi_{s}\left(\theta_{j}\right)=\beta_{j} \theta_{j}^{\beta_{j}-1} d_{j}^{-\beta_{j}}, \quad 0<\theta_{j}<d_{j} ; d_{j}, \beta_{j}>0 .
$$

We consider the prior density function of $\lambda$ as follows:

$$
\pi_{s}(\lambda)=A \lambda^{a-1} \exp \{-b \lambda\}, \quad a, b>0, \lambda>1,
$$

where $A$ is the regularization factor.
Then the marginal posterior density functions of the parameters $\lambda, \theta_{1}$, and $\theta_{2}$ are, respectively,

$$
\begin{aligned}
& \pi_{s}(\lambda \mid t) \propto \lambda^{m-N_{1}+a-1} \\
& \cdot \exp \left\{-\left[\left(\alpha_{1}+\alpha_{2}\right)\left(\ln W_{1}-\ln W_{2}\right)+b\right] \lambda\right\}, \\
& \quad a, b>0, \lambda>1, \\
& \pi_{s}\left(\theta_{j} \mid t\right)=\frac{\left(\alpha_{j} n+\beta_{j}\right) \theta_{j}^{\alpha_{j} n+\beta_{j}-1}}{d_{j}^{*\left(\alpha_{j} n+\beta_{j}\right)}}, \\
& 0<\theta_{j}<d_{j}^{*}, d_{j}^{*}=\min \left(t_{j 1}, d_{j}\right),
\end{aligned}
$$

where $t_{j 1}$ is the first-failure time caused by the $j$ th failure cause $(j=1,2)$.

Under the squared loss function, the Bayesian estimate of $\theta_{j}(j=1,2)$ can be easily obtained as

$$
\widehat{\theta}_{j \mathrm{BS}}=\int_{0}^{d_{j}^{*}} \theta_{j} \pi_{S}\left(\theta_{j} \mid t\right) d \theta_{j}=\frac{\left(\alpha_{j} n+\beta_{j}\right) d_{j}^{*}}{\left(\alpha_{j} n+\beta_{j}+1\right)} .
$$

To obtain the Bayesian estimate of $\lambda$, we expand $H(\lambda)=$ $\lambda^{m-N_{1}+a}$ and $h(\lambda)=\lambda^{m-N_{1}+a-1}$ in Taylor series around the maximum likelihood estimate $\hat{\lambda}$ to get the following approximation:

$$
\begin{gathered}
H(\lambda) \approx H(\widehat{\lambda})+H^{\prime}(\widehat{\lambda})(\lambda-\hat{\lambda}), \\
h(\lambda) \approx h(\widehat{\lambda})+h^{\prime}(\widehat{\lambda})(\lambda-\widehat{\lambda}),
\end{gathered}
$$

where $H^{\prime}(\lambda)=\left(m-N_{1}+a\right) \lambda^{m-N_{1}+a-1}$ and $h^{\prime}(\lambda)=\left(m-N_{1}+\right.$ $a-1) \lambda^{m-N_{1}+a-2}$. 
Therefore, under the squared loss function, the Bayesian estimate of $\lambda$ can be approximated as

$$
\widehat{\lambda}_{\mathrm{BS}}=\frac{H(\widehat{\lambda})+H^{\prime}(\widehat{\lambda})\left(1+I_{1}^{-1}-\widehat{\lambda}\right)}{h(\widehat{\lambda})+h^{\prime}(\widehat{\lambda})\left(1+I_{1}^{-1}-\widehat{\lambda}\right)},
$$

where $I_{1}=\left(\alpha_{1}+\alpha_{2}\right)\left(\ln W_{1}-\ln W_{2}\right)+b$.

3.3. E-Bayesian Estimates. By assuming that the hyperparameters follow certain distributions, the Bayesian estimates can be obtained when the hyperparameters in prior distributions are unknown. However, complicated integration is often a hard work. Han $[16,17]$ gave the definition of E-Bayesian estimation to overcome this problem. So we will derive the E-Bayesian estimates of the unknown parameters and acceleration factor in this subsection.

Suppose that the hyperparameters $a$ and $b$ and $\beta_{j}$ and $d_{j}(j=1,2)$ have the joint prior density functions $\pi_{e}(a, b)$ and $\pi_{e}\left(\beta_{j}, d_{j}\right)$, respectively. Then the E-Bayesian estimates of $\lambda$ and $\theta_{j}(j=1,2)$ are

$$
\begin{aligned}
\hat{\lambda}_{\mathrm{EB}} & =\iint_{D} \hat{\lambda}_{\mathrm{BS}} \pi_{e}(a, b) d a d b, \\
\widehat{\theta}_{j \mathrm{~EB}} & =\iint_{D} \hat{\theta}_{j \mathrm{BS}} \pi_{e}\left(\beta_{j}, d_{j}\right) d \beta_{j} d\left(d_{j}\right),
\end{aligned}
$$

where $\hat{\lambda}_{\mathrm{BS}}$ and $\widehat{\theta}_{j \mathrm{BS}}$ are the Bayesian estimates of $\lambda$ and $\theta_{j}(j=$ $1,2)$, respectively.

According to Han [16], $a$ and $b$ and $\beta_{j}$ and $d_{j}(j=1,2)$ should be selected to guarantee that $\pi_{s}(\lambda)$ and $\pi_{s}\left(\theta_{j}\right)$ are decreasing functions of $\lambda$ and $\theta_{j}$, respectively. The derivatives of $\pi_{s}(\lambda)$ with respect to $\lambda$ and $\pi_{s}\left(\theta_{j}\right)$ with respect to $\theta_{j}$ are

$$
\begin{aligned}
& \frac{\partial \pi_{s}(\lambda)}{\partial \lambda} \propto \lambda^{a-2} \exp \{-b \lambda\}(a-1-b \lambda), \\
& \frac{\partial \pi_{s}\left(\theta_{j}\right)}{\partial \theta_{j}}=\beta_{j}\left(\beta_{j}-1\right) \theta_{j}^{\beta_{j}-2} d_{j}^{-\beta_{j}},
\end{aligned}
$$

When $0<a<1, b>0, \partial \pi_{s}(\lambda) / \partial \lambda<0$, and when $0<\beta_{j}<1$, $d_{j}>0, \partial \pi_{s}\left(\theta_{j}\right) / \partial \theta_{j}<0$. Thus, $\pi_{s}(\lambda)$ and $\pi_{s}\left(\theta_{j}\right)$ are decreasing functions of $\lambda$ and $\theta_{j}$, respectively.

Given that $0<a<1$ and $0<\beta_{j}<1$, the larger $b$ and $d_{j}$ are, the thinner the tail of $\pi_{s}(\lambda)$ and $\pi_{s}\left(\theta_{j}\right)$ will be. Berger [24] showed that the thinner tailed prior distribution often reduces the robustness of the Bayesian estimate. Consequently, $b$ and $d_{j}(j=1,2)$ should be given upper bounds $l$ and $h_{j}^{\prime}$, respectively. We consider the following two joint prior distributions of $a$ and $b$ and $\beta_{j}$ and $d_{j}(j=1,2)$, respectively:

$$
\begin{gathered}
\pi_{1}(a, b)=\frac{2 b}{l^{2}}, \quad 0<a<1, \quad 0<b<l, \\
\pi_{2}(a, b)=\frac{1}{l}, \quad 0<a<1, \quad 0<b<l, \\
\pi_{1 j}\left(\beta_{j}, d_{j}\right)=\frac{2 d_{j}}{h_{j}^{\prime 2}}, \quad 0<\beta_{j}<1,0<d_{j}<h_{j}^{\prime}, \\
\pi_{2 j}\left(\beta_{j}, d_{j}\right)=\frac{1}{h_{j}^{\prime}}, \quad 0<\beta_{j}<1, \quad 0<d_{j}<h_{j}^{\prime} .
\end{gathered}
$$

From (19), (20), and (23), we derive the E-Bayesian estimates of $\lambda$ as

$$
\begin{aligned}
& \hat{\lambda}_{\mathrm{EB} 1}=\frac{2}{l^{2}} \int_{0}^{l} \int_{0}^{1} \frac{b\left[H(\widehat{\lambda})+H^{\prime}(\widehat{\lambda})\left(1+I_{2}^{-1}-\widehat{\lambda}\right)\right]}{h(\widehat{\lambda})+h^{\prime}(\hat{\lambda})\left(1+I_{2}^{-1}-\hat{\lambda}\right)} d a d b \\
& =\frac{2 \hat{\lambda}}{l^{2}} \int_{0}^{l} b \int_{0}^{1} \frac{\left(I_{1}+b\right) \hat{\lambda}+\left(m-N_{1}+a\right)\left[1+\left(I_{1}+b\right)(1-\hat{\lambda})\right]}{\left(I_{1}+b\right) \hat{\lambda}+\left(m-N_{1}+a-1\right)\left[1+\left(I_{1}+b\right)(1-\hat{\lambda})\right]} d a d b=\hat{\lambda}+\frac{\hat{\lambda}}{l^{2}}\left(Q_{1}-Q_{2}\right), \\
& \widehat{\lambda}_{\mathrm{EB} 2}=\frac{1}{l} \int_{0}^{l} \int_{0}^{1} \frac{H(\widehat{\lambda})+H^{\prime}(\widehat{\lambda})\left(1+I_{2}^{-1}-\widehat{\lambda}\right)}{h(\widehat{\lambda})+h^{\prime}(\widehat{\lambda})\left(1+I_{2}^{-1}-\hat{\lambda}\right)} d a d b \\
& =\frac{\hat{\lambda}}{l} \int_{0}^{l} \int_{0}^{1} \frac{\left(I_{1}+b\right) \hat{\lambda}+\left(m-N_{1}+a\right)\left[1+\left(I_{1}+b\right)(1-\hat{\lambda})\right]}{\left(I_{1}+b\right) \hat{\lambda}+\left(m-N_{1}+a-1\right)\left[1+\left(I_{1}+b\right)(1-\hat{\lambda})\right]} d a d b=\hat{\lambda}+\frac{\hat{\lambda}}{l}\left(E_{1}-E_{2}\right),
\end{aligned}
$$

where

$$
\begin{aligned}
Q_{1} & =\left[l^{2}-\left(I_{1}+\frac{N_{2}}{z(\widehat{\lambda})}\right)^{2}\right] \ln \left[\left(I_{1}+l\right) z(\widehat{\lambda})+N_{2}\right] \\
& +\left(I_{1}+\frac{N_{2}}{z(\hat{\lambda})}\right)^{2} \ln \left(I_{1} z(\widehat{\lambda})+N_{2}\right)+\frac{N_{2} l}{z(\widehat{\lambda})}
\end{aligned}
$$$$
Q_{2}=\left[l^{2}-\left(I_{1}+\frac{N_{2}-1}{p(\bar{\lambda})}\right)^{2}\right]
$$$$
\cdot \ln \left[\left(I_{1}+l\right) p(\bar{\lambda})+N_{2}-1\right]+\left(I_{1}+\frac{N_{2}-1}{p(\bar{\lambda})}\right)^{2}
$$ 


$$
\begin{aligned}
& \cdot \ln \left(I_{1} p(\widehat{\lambda})+N_{2}-1\right)+\frac{\left(N_{2}-1\right) l}{p(\bar{\lambda})}, \\
& E_{1}=\left(I_{1}+l+\frac{N_{2}}{z(\widehat{\lambda})}\right) \ln \left[\left(I_{1}+l\right) z(\widehat{\lambda})+N_{2}\right] \\
& -\left(I_{1}+\frac{N_{2}}{z(\widehat{\lambda})}\right) \ln \left[I_{1} z(\widehat{\lambda})+N_{2}\right], \\
& E_{2}=\left(I_{1}+l+\frac{N_{2}-1}{p(\hat{\lambda})}\right) \ln \left[\left(I_{1}+l\right) p(\widehat{\lambda})+N_{2}-1\right] \\
& -\left(I_{1}+\frac{N_{2}-1}{p(\widehat{\lambda})}\right) \ln \left[I_{1} p(\widehat{\lambda})+N_{2}-1\right], \\
& z(\hat{\lambda})=\hat{\lambda}+N_{2}(1-\hat{\lambda}), \\
& p(\widehat{\lambda})=\widehat{\lambda}+\left(N_{2}-1\right)(1-\hat{\lambda}) \text {, } \\
& N_{2}=m-N_{1} \text {. }
\end{aligned}
$$

Similarly, from (17), (21), and (24), the E-Bayesian estimate of $\theta_{j}(j=1,2)$ can be obtained as

$$
\begin{gathered}
\widehat{\theta}_{j \mathrm{~EB} 1}= \begin{cases}\frac{2 h_{j}^{\prime}}{3}\left(1-\ln \frac{\alpha_{j} n+2}{\alpha_{j} n+1}\right), & d_{j}^{*}=d_{j}, \\
d_{j}^{*}\left(1-\ln \frac{\alpha_{j} n+2}{\alpha_{j} n+1}\right), & d_{j}^{*} \neq d_{j},\end{cases} \\
\widehat{\theta}_{j \mathrm{~EB} 2}= \begin{cases}\frac{h_{j}^{\prime}}{2}\left(1-\ln \frac{\alpha_{j} n+2}{\alpha_{j} n+1}\right), & d_{j}^{*}=d_{j}, \\
d_{j}^{*}\left(1-\ln \frac{\alpha_{j} n+2}{\alpha_{j} n+1}\right), & d_{j}^{*} \neq d_{j} .\end{cases}
\end{gathered}
$$

\section{Application of the Pareto Distribution}

In this section, we analyze two real data sets to illustrate the application of the Pareto distribution in engineering practice. The first data set (Set I) represent the failure time of 20 mechanical components. These data are taken from Murthy et al. [25] and have been used by Bourguignon et al. [22]. These data are $0.067,0.068,0.076,0.081,0.084,0.085,0.085$, $0.086,0.089,0.098,0.098,0.114,0.114,0.115,0.121,0.125$, $0.131,0.149,0.160$, and 0.485 . The second data set (Set II) is given by Nelson [26], which represents the failure times to breakdown of a type of electronic insulating material subject to a constant-voltage stress. These data are $0.35,0.59,0.96$, $0.99,1.69,1.97,2.07,2.58,2.71,2.90,3.67,3.99,5.35,13.77$, and 25.50 .

We check the validity for the Pareto distribution $P(\alpha, \theta)$ by using the Kolmogorov test. The statistics of the Kolmogorov test are $D_{n}=\sup _{x}\left|F(x)-F_{n}(x)\right|=\max _{1 \leq i \leq 20} \mid F\left(x_{i}\right)-$ $F_{n}\left(x_{i}\right) \mid$, where $F(x)$ is the distribution function of $P(\alpha, \theta)$ and $F(x)=1-(\theta / x)^{\alpha}, x>0$, and $F_{n}(x)$ is the empirical distribution functions constructed based on the above data Set I and Set II.
For the data Set I, when the parameters $\alpha=2.113$ and $\theta=0.067$, the observation value of the statistic is $\widehat{D}_{n}=$ $\max _{1 \leq i \leq 20}\left|F\left(x_{i}\right)-F_{n}\left(x_{i}\right)\right|=0.1303$. For $n=20$, we choose the value of confidence level $\alpha$ as $\alpha=0.01,0.02,0.05$, respectively. By using the table of critical value on the Kolmogorov test, we get the critical value $\widehat{D}_{n, \alpha}$, where $\widehat{D}_{20,0.05}=0.29403, \widehat{D}_{20,0.02}=$ 0.32266, and $\widehat{D}_{20,0.01}=0.35241$. Since $\widehat{D}_{n}<\widehat{D}_{20,0.05}, \widehat{D}_{n}<$ $\widehat{D}_{20,0.02}$, and $\widehat{D}_{n}<\widehat{D}_{20,0.01}$, thus, the fit of Pareto distribution $P(\alpha, \theta)=P(2.113,0.067)$ to the above data Set I is reasonable.

For data Set II, when the parameters $\alpha=0.51$ and $\theta=0.35$, the observation value of the statistic is $\widehat{D}_{n}=$ $\max _{1 \leq i \leq 20}\left|F\left(x_{i}\right)-F_{n}\left(x_{i}\right)\right|=0.2187$. For $n=15$, we choose the value of confidence level $\alpha$ as $\alpha=0.01,0.02,0.05$, respectively. By using the table of critical value on the Kolmogorov test, we get the critical value $\widehat{D}_{n, \alpha}$, where $\widehat{D}_{15,0.05}=0.33760$, $\widehat{D}_{15,0.02}=0.37713$, and $\widehat{D}_{15,0.01}=0.40120$. Since $\widehat{D}_{n}<$ $\widehat{D}_{15,0.05}, \widehat{D}_{n}<\widehat{D}_{15,0.02}$, and $\widehat{D}_{n}<\widehat{D}_{15,0.01}$, the fit of Pareto distribution $P(\alpha, \theta)=P(0.51,0.35)$ to the above data Set II is reasonable. Therefore, the use of the Pareto distribution is well justified.

\section{Numerical Example}

In this section, we present numerical example using Monte Carlo simulations to illustrate the estimation procedure presented in this paper. The performance of three types of Bayesian estimates for different sample sizes and different progressive censoring schemes (PCS) is assessed based on simulation data. The term "different PCS" means different sets of $R_{i}$ 's. In this paper, we consider the following three different PCS.

Scheme 1. $R_{1}=\cdots=R_{m-1}=0$, and $R_{m}=n-m$.

Scheme 2. $R_{1}=R_{2}=\cdots=R_{L}=0, R_{L+1}=\cdots=R_{m-1}=1$, and $R_{m}=n-2 m+1+L$.

Scheme 3. $R_{1}=R_{2}=\cdots=R_{L+1}=1, R_{L+2}=\cdots=R_{m-1}=$ 0 , and $R_{m}=n-m-1-L$, where $L=[m / 4]+1,[m / 4]$ representing the maximum integer that does not exceed $m / 4$, $m \geq 10$

Before progressing further, we first describe how to generate Type II progressively censored competing failure data from Pareto distribution under SSPALT.

Step 1. Given $m, n$, and a certain censoring scheme, generate two samples $U_{1}, U_{2}, \ldots, U_{m}$ and $W_{1}, W_{2}, \ldots, W_{m}$ from the uniform $(0,1)$ distribution [27].

Step 2. Given $\alpha_{j}, \theta_{j}(j=1,2)$, compute $t_{11 i}$ and $t_{12 i}$ by solving equations $U_{i}=1-\theta_{1}^{\alpha_{1}} t_{11 i}^{-\alpha_{1}}$ and $W_{i}=1-\theta_{2}^{\alpha_{2}} t_{12 i}^{-\alpha_{2}}$, respectively. Then record the minimum of $\left(t_{11 i}, t_{12 i}\right)$ as $t_{i}^{*}$ and the corresponding index of the minimum (if $t_{11 i}<t_{12 i}$, set $c_{i}^{*}=1$; else set $\left.c_{i}^{*}=2\right)$ for $1 \leq i \leq m$.

Step 3. For prefixed stress changing time $\tau$, find $N_{1}$ such that $t_{N_{1}}^{*} \leq \tau<t_{N_{1}+1}^{*}$. Then let $t_{i}=t_{i}^{*}$ and $c_{i}=c_{i}^{*}$ for $1 \leq i \leq N_{1}$. 
TABLE 1: The AREs and MSEs of all estimates for different $m, n$ and for censoring Scheme 1.

\begin{tabular}{|c|c|c|c|c|c|}
\hline$(n, m)$ & Para. & $\begin{array}{c}\text { OBE } \\
\text { ARE (MSE) } \\
\end{array}$ & $\begin{array}{c}\text { BE } \\
\text { ARE (MSE) }\end{array}$ & $\begin{array}{c}\text { E-BE1 } \\
\text { ARE (MSE) }\end{array}$ & $\begin{array}{c}\text { E-BE2 } \\
\text { ARE (MSE) }\end{array}$ \\
\hline \multirow{3}{*}{$(40,10)$} & $\lambda$ & $0.0526(0.0073)$ & $0.0570(0.0081)$ & $0.0519(0.0052)$ & $0.0498(0.050)$ \\
\hline & $\theta_{1}$ & $0.0360(0.0048)$ & $0.0378(0.0076)$ & $0.0392(0.0075)$ & $0.0382(0.0065)$ \\
\hline & $\theta_{2}$ & $0.0474(0.0115)$ & $0.0485(0.0138)$ & $0.0512(0.0128)$ & $0.0508(0.0107)$ \\
\hline \multirow{3}{*}{$(40,15)$} & $\lambda$ & $0.0514(0.0069)$ & $0.0551(0.0067)$ & $0.0515(0.0048)$ & $0.0495(0.0044)$ \\
\hline & $\theta_{1}$ & $0.0353(0.0042)$ & $0.0364(0.0065)$ & $0.0387(0.0062)$ & $0.0374(0.0061)$ \\
\hline & $\theta_{2}$ & $0.0462(0.0107)$ & $0.0472(0.0120)$ & $0.0504(0.0112)$ & $0.0500(0.0099)$ \\
\hline \multirow{3}{*}{$(40,20)$} & $\lambda$ & $0.0489(0.0057)$ & $0.0548(0.0059)$ & $0.0505(0.0044)$ & $0.0495(0.0041)$ \\
\hline & $\theta_{1}$ & $0.0336(0.0032)$ & $0.0341(0.0056)$ & $0.0372(0.0051)$ & $0.0365(0.0049)$ \\
\hline & $\theta_{2}$ & $0.0458(0.0104)$ & $0.0463(0.0113)$ & $0.0500(0.0110)$ & $0.0491(0.0095)$ \\
\hline \multirow{3}{*}{$(45,10)$} & $\lambda$ & $0.0513(0.0070)$ & $0.0564(0.0080)$ & $0.0514(0.0050)$ & $0.0498(0.0048)$ \\
\hline & $\theta_{1}$ & $0.0357(0.0046)$ & $0.0375(0.0074)$ & $0.0390(0.0071)$ & $0.0383(0.0063)$ \\
\hline & $\theta_{2}$ & $0.0471(0.0111)$ & $0.0486(0.0127)$ & $0.0505(0.0115)$ & $0.0512(0.0100)$ \\
\hline \multirow{3}{*}{$(45,15)$} & $\lambda$ & $0.0510(0.0064)$ & $0.0549(0.0058)$ & $0.0514(0.0047)$ & $0.0491(0.0046)$ \\
\hline & $\theta_{1}$ & $0.0350(0.0040)$ & $0.0362(0.0061)$ & $0.0386(0.0059)$ & $0.0370(0.0060)$ \\
\hline & $\theta_{2}$ & $0.0460(0.0101)$ & $0.0470(0.0115)$ & $0.0501(0.0110)$ & $0.0500(0.0098)$ \\
\hline \multirow{3}{*}{$(45,20)$} & $\lambda$ & $0.0484(0.0056)$ & $0.0542(0.0054)$ & $0.0504(0.0041)$ & $0.0493(0.0038)$ \\
\hline & $\theta_{1}$ & $0.0334(0.0030)$ & $0.0338(0.0051)$ & $0.0370(0.0048)$ & $0.0362(0.0048)$ \\
\hline & $\theta_{2}$ & $0.0454(0.0100)$ & $0.0462(0.0111)$ & $0.0497(0.0105)$ & $0.0489(0.0087)$ \\
\hline \multirow{3}{*}{$(50,10)$} & $\lambda$ & $0.0511(0.0068)$ & $0.0563(0.0076)$ & $0.0511(0.0047)$ & $0.0494(0.0046)$ \\
\hline & $\theta_{1}$ & $0.0353(0.0044)$ & $0.0373(0.0071)$ & $0.0387(0.0070)$ & $0.0380(0.0061)$ \\
\hline & $\theta_{2}$ & $0.0468(0.0109)$ & $0.0483(0.0118)$ & $0.0502(0.0112)$ & $0.0510(0.0094)$ \\
\hline \multirow{3}{*}{$(50,15)$} & $\lambda$ & $0.0509(0.0062)$ & $0.0546(0.0058)$ & $0.0512(0.0045)$ & $0.0492(0.0045)$ \\
\hline & $\theta_{1}$ & $0.0348(0.0037)$ & $0.0359(0.0060)$ & $0.0382(0.0058)$ & $0.0368(0.0058)$ \\
\hline & $\theta_{2}$ & $0.0459(0.0095)$ & $0.0468(0.0110)$ & $0.0500(0.0107)$ & $0.0500(0.0093)$ \\
\hline \multirow{3}{*}{$(50,20)$} & $\lambda$ & $0.0481(0.0054)$ & $0.0541(0.0051)$ & $0.0503(0.0040)$ & $0.0491(0.0035)$ \\
\hline & $\theta_{1}$ & $0.0330(0.0027)$ & $0.0335(0.0047)$ & $0.0368(0.0042)$ & $0.0369(0.0043)$ \\
\hline & $\theta_{2}$ & $0.0445(0.0096)$ & $0.0462(0.0106)$ & $0.0497(0.0102)$ & $0.0488(0.0072)$ \\
\hline
\end{tabular}

Step 4. For $N_{1}+1 \leq i \leq m$, generate $t_{21 i}$ by solving the equation $U_{i}=1-\theta_{1}^{\alpha_{1}} \tau^{\alpha_{1}(\lambda-1)} t_{21 i}^{-\alpha_{1} \lambda}$, and generate $t_{22 i}$ by solving $W_{i}=1-\theta_{2}^{\alpha_{2}} \tau^{\alpha_{2}(\lambda-1)} t_{22 i}^{-\alpha_{2} \lambda}$. Again, take the minimum of $\left(t_{21 i}, t_{22 i}\right)$ as $t_{i}^{*}$ as well as the corresponding index of the minimum (if $t_{21 i}<t_{22 i}$, set $c_{i}^{*}=1$; else set $c_{i}^{*}=2$ ).

Step 5. For $N_{1}+1 \leq i \leq m$, let $t_{i}=t_{i}^{*}$ and $c_{i}=c_{i}^{*}$.

Then $\left\{\left(t_{1}, c_{1}, R_{1}\right), \ldots,\left(t_{m}, c_{m}, R_{m}\right)\right\}$ is the required sample.

We assumed that the occurrence time of the $j$ th $(j=1,2)$ failure cause of tested product follows Pareto distributions $P(\alpha, \theta)=P(2.113,0.067)$ and $P(\alpha, \theta)=P(1.82,0.051)$ under use stress levels $S_{0}$, and $\lambda=2.0$ and $\tau=0.11$, the parameters of prior density are taken as $a=0.8, b=2.0, \beta_{1}=0.85$, $\beta_{2}=0.6, d_{1}=1.8$, and $d_{2}=2.1$. Considering different $m, n$ and censoring schemes, we generate samples according to the above steps. We replicate the process of evaluating 1000 times in each case and compute the average relative errors (AREs) and mean squared errors (MSEs). ARE and MSE of the estimation $\hat{\theta}$ of the parameter $\theta$ can be calculated according to the following formulas:

$$
\begin{aligned}
& \operatorname{ARE}(\widehat{\theta})=\frac{1}{1000} \sum_{k=1}^{1000} \frac{\left|\theta-\widehat{\theta}^{(k)}\right|}{\theta}, \\
& \operatorname{MSE}(\widehat{\theta})=\frac{1}{1000} \sum_{k=1}^{1000}\left(\theta-\widehat{\theta}^{(k)}\right)^{2},
\end{aligned}
$$

where $\widehat{\theta}^{(k)}$ is the $k$ th estimator for the parameter $\theta$.

We calculate the AREs and MSEs of the objective Bayesian estimates (OBE), Bayesian estimates (BE), and E-Bayesian estimates (E-BE), respectively. The simulation results are presented in Tables 1-3. In Tables 1-3, we denote the E-BE based on the first prior as E-BE1 and the E-BE based on the second prior as E-BE2.

From Tables 1-3, the following observations can be made:

(1) For fixed $n$, as $m$ increases the AREs and MSEs decrease for all estimates.

(2) In terms of the values of AREs and MSEs, the OBE performances are better than that of $\mathrm{BE}$, and $\mathrm{E}-\mathrm{BE} 2$ 
TABLE 2: The AREs and MSEs of all estimates for different $m, n$ and for censoring Scheme 2.

\begin{tabular}{|c|c|c|c|c|c|}
\hline$(n, m)$ & Para. & $\begin{array}{c}\text { OBE } \\
\text { ARE (MSE) } \\
\end{array}$ & $\begin{array}{c}\text { BE } \\
\text { ARE (MSE) }\end{array}$ & $\begin{array}{c}\text { E-BE1 } \\
\text { ARE (MSE) }\end{array}$ & $\begin{array}{c}\text { E-BE2 } \\
\text { ARE (MSE) }\end{array}$ \\
\hline \multirow{3}{*}{$(40,10)$} & $\lambda$ & $0.0531(0.0080)$ & $0.0576(0.0084)$ & $0.0521(0.0055)$ & $0.0501(0.0053)$ \\
\hline & $\theta_{1}$ & $0.0365(0.0051)$ & $0.0381(0.0081)$ & $0.0398(0.0079)$ & $0.0386(0.0067)$ \\
\hline & $\theta_{2}$ & $0.0478(0.0118)$ & $0.0488(0.0142)$ & $0.0511(0.0125)$ & $0.0516(0.0117)$ \\
\hline \multirow{3}{*}{$(40,15)$} & $\lambda$ & $0.0516(0.0071)$ & $0.0551(0.0082)$ & $0.0515(0.0051)$ & $0.0495(0.0049)$ \\
\hline & $\theta_{1}$ & $0.0357(0.0046)$ & $0.0364(0.0080)$ & $0.0387(0.0064)$ & $0.0374(0.0061)$ \\
\hline & $\theta_{2}$ & $0.0465(0.0113)$ & $0.0472(0.0138)$ & $0.0504(0.0114)$ & $0.0500(0.0112)$ \\
\hline \multirow{3}{*}{$(40,20)$} & $\lambda$ & $0.0489(0.0077)$ & $0.0548(0.0078)$ & $0.0505(0.0058)$ & $0.0495(0.0056)$ \\
\hline & $\theta_{1}$ & $0.0336(0.0044)$ & $0.0341(0.0075)$ & $0.0372(0.0056)$ & $0.0365(0.0053)$ \\
\hline & $\theta_{2}$ & $0.0458(0.0110)$ & $0.0463(0.0133)$ & $0.0500(0.0112)$ & $0.0491(0.0110)$ \\
\hline \multirow{3}{*}{$(45,10)$} & $\lambda$ & $0.0527(0.0079)$ & $0.0564(0.0079)$ & $0.0511(0.0052)$ & $0.0501(0.0051)$ \\
\hline & $\theta_{1}$ & $0.0365(0.0048)$ & $0.0380(0.0077)$ & $0.0394(0.0075)$ & $0.0382(0.0065)$ \\
\hline & $\theta_{2}$ & $0.0474(0.0115)$ & $0.0485(0.0133)$ & $0.0509(0.0122)$ & $0.0512(0.0114)$ \\
\hline \multirow{3}{*}{$(45,15)$} & $\lambda$ & $0.0513(0.0067)$ & $0.0549(0.0075)$ & $0.0513(0.0050)$ & $0.0492(0.0048)$ \\
\hline & $\theta_{1}$ & $0.0348(0.0037)$ & $0.0364(0.0074)$ & $0.0382(0.0062)$ & $0.0370(0.0058)$ \\
\hline & $\theta_{2}$ & $0.0464(0.0112)$ & $0.0470(0.0131)$ & $0.0501(0.0108)$ & $0.0487(0.0107)$ \\
\hline \multirow{3}{*}{$(45,20)$} & $\lambda$ & $0.0486(0.0065)$ & $0.0546(0.0071)$ & $0.0501(0.0057)$ & $0.0492(0.0045)$ \\
\hline & $\theta_{1}$ & $0.0332(0.0034)$ & $0.0341(0.0070)$ & $0.0372(0.0056)$ & $0.0365(0.0051)$ \\
\hline & $\theta_{2}$ & $0.0451(0.0110)$ & $0.0457(0.0127)$ & $0.0492(0.0104)$ & $0.0490(0.0104)$ \\
\hline \multirow{3}{*}{$(50,10)$} & $\lambda$ & $0.0521(0.0075)$ & $0.0560(0.0076)$ & $0.0508(0.0046)$ & $0.0500(0.0043)$ \\
\hline & $\theta_{1}$ & $0.0362(0.0046)$ & $0.0380(0.0075)$ & $0.0392(0.0074)$ & $0.0381(0.0063)$ \\
\hline & $\theta_{2}$ & $0.0471(0.0113)$ & $0.0483(0.0131)$ & $0.0504(0.0120)$ & $0.0510(0.0112)$ \\
\hline \multirow{3}{*}{$(50,15)$} & $\lambda$ & $0.0504(0.0065)$ & $0.0546(0.0074)$ & $0.0512(0.0050)$ & $0.0491(0.0045)$ \\
\hline & $\theta_{1}$ & $0.0354(0.0036)$ & $0.0364(0.0072)$ & $0.0382(0.0061)$ & $0.0370(0.0055)$ \\
\hline & $\theta_{2}$ & $0.0464(0.0110)$ & $0.0470(0.0130)$ & $0.0501(0.0105)$ & $0.0496(0.0104)$ \\
\hline \multirow{3}{*}{$(50,20)$} & $\lambda$ & $0.0475(0.0059)$ & $0.0536(0.0072)$ & $0.0491(0.0048)$ & $0.0487(0.0044)$ \\
\hline & $\theta_{1}$ & $0.0324(0.0029)$ & $0.0332(0.0170)$ & $0.0366(0.0052)$ & $0.0356(0.0051)$ \\
\hline & $\theta_{2}$ & $0.0443(0.0107)$ & $0.0450(0.0127)$ & $0.0483(0.0102)$ & $0.0481(0.0100)$ \\
\hline
\end{tabular}

performances are better than that of E-BE1 under the different progressive censoring schemes.

(3) Under the different progressive censoring schemes, for all estimates, the values of AREs are less than 0.06, and the values of MSEs are less than 0.015. Hence the results of all estimates are satisfied.

\section{Conclusions}

In this paper, the Bayesian analysis for the step-stress partially accelerated competing failure model from Pareto distribution is investigated under Type II progressive censoring. Based on the noninformative prior and informative prior distributions, we derive the objective Bayesian estimates and Bayesian estimates of the unknown parameters and acceleration factor, respectively. Considering such case that the hyperparameters are unknown and follow certain distributions, the E-Bayesian method is utilized to estimate the unknown parameters and acceleration factor. We analyze two real data sets to illustrate the application of the Pareto distribution in engineering practice. Finally, the comparisons of the efficiencies of the objective Bayesian estimates, Bayesian estimates, and corresponding E-Bayesian estimates are conducted. It is concluded that the OBE performances are better than that of $\mathrm{BE}$ and E-BE2 performances are better than that of E-BE1 under the different progressive censoring schemes. In addition, we discuss the effects of sample sizes and different progressive censoring schemes on the estimates. The simulation results demonstrate that for fixed $n$ the AREs and MSEs of all estimates decrease with $m$ increasing.

\section{Competing Interests}

The authors declare that they have no competing interests.

\section{Acknowledgments}

The work is supported by the National Natural Science Foundation of China (71401134, 71571144, and 71171164), The Natural Science Basic Research Program of Shaanxi Province (2015JM1003), and The International Cooperation 
TABLE 3: The AREs and MSEs of all estimates for different $m, n$ and for censoring Scheme 3.

\begin{tabular}{|c|c|c|c|c|c|}
\hline$(n, m)$ & Para. & $\begin{array}{c}\text { OBE } \\
\text { ARE (MSE) }\end{array}$ & $\begin{array}{c}\text { BE } \\
\text { ARE (MSE) }\end{array}$ & $\begin{array}{c}\text { E-BE1 } \\
\text { ARE (MSE) }\end{array}$ & $\begin{array}{c}\text { E-BE2 } \\
\text { ARE (MSE) }\end{array}$ \\
\hline \multirow{3}{*}{$(40,10)$} & $\lambda$ & $0.0535(0.0079)$ & $0.0578(0.0082)$ & $0.0521(0.0056)$ & $0.0510(0.0054)$ \\
\hline & $\theta_{1}$ & $0.0358(0.0053)$ & $0.0385(0.0083)$ & $0.0399(0.0082)$ & $0.0390(0.0072)$ \\
\hline & $\theta_{2}$ & $0.0481(0.0129)$ & $0.0491(0.0145)$ & $0.0512(0.0130)$ & $0.0520(0.0122)$ \\
\hline \multirow{3}{*}{$(40,15)$} & $\lambda$ & $0.0523(0.0072)$ & $0.0551(0.0080)$ & $0.0520(0.0052)$ & $0.0501(0.0051)$ \\
\hline & $\theta_{1}$ & $0.0364(0.0048)$ & $0.0371(0.0077)$ & $0.0396(0.0076)$ & $0.0380(0.0071)$ \\
\hline & $\theta_{2}$ & $0.0471(0.0122)$ & $0.0480(0.0142)$ & $0.0511(0.0129)$ & $0.0512(0.0120)$ \\
\hline \multirow{3}{*}{$(40,20)$} & $\lambda$ & $0.0491(0.0066)$ & $0.0552(0.0078)$ & $0.0513(0.0051)$ & $0.0504(0.0050)$ \\
\hline & $\theta_{1}$ & $0.0342(0.0043)$ & $0.0350(0.0075)$ & $0.0381(0.0071)$ & $0.0373(0.0068)$ \\
\hline & $\theta_{2}$ & $0.0465(0.0120)$ & $0.0469(0.0140)$ & $0.0507(0.0124)$ & $0.0499(0.0118)$ \\
\hline \multirow{3}{*}{$(45,10)$} & $\lambda$ & $0.0532(0.0075)$ & $0.0571(0.0079)$ & $0.0521(0.0052)$ & $0.0504(0.0049)$ \\
\hline & $\theta_{1}$ & $0.0363(0.0051)$ & $0.0381(0.0081)$ & $0.0398(0.0081)$ & $0.0390(0.0067)$ \\
\hline & $\theta_{2}$ & $0.0480(0.0127)$ & $0.0491(0.0141)$ & $0.0512(0.0128)$ & $0.0520(0.0120)$ \\
\hline \multirow{3}{*}{$(45,15)$} & $\lambda$ & $0.0518(0.0068)$ & $0.0551(0.0075)$ & $0.0520(0.0050)$ & $0.0501(0.0046)$ \\
\hline & $\theta_{1}$ & $0.0358(0.0050)$ & $0.0371(0.0075)$ & $0.0391(0.0076)$ & $0.0381(0.0064)$ \\
\hline & $\theta_{2}$ & $0.0467(0.0118)$ & $0.0478(0.0137)$ & $0.0510(0.0123)$ & $0.0510(0.0117)$ \\
\hline \multirow{3}{*}{$(45,20)$} & $\lambda$ & $0.0491(0.0060)$ & $0.0552(0.0072)$ & $0.0513(0.0047)$ & $0.0450(0.0042)$ \\
\hline & $\theta_{1}$ & $0.0340(0.0048)$ & $0.0345(0.0069)$ & $0.0381(0.0065)$ & $0.0371(0.0062)$ \\
\hline & $\theta_{2}$ & $0.0462(0.0115)$ & $0.0470(0.0129)$ & $0.0503(0.0120)$ & $0.0493(0.0113)$ \\
\hline \multirow{3}{*}{$(50,10)$} & $\lambda$ & $0.0530(0.0074)$ & $0.0571(0.0067)$ & $0.0520(0.0050)$ & $0.0502(0.0045)$ \\
\hline & $\theta_{1}$ & $0.0353(0.0050)$ & $0.0373(0.0078)$ & $0.0387(0.0079)$ & $0.0380(0.0064)$ \\
\hline & $\theta_{2}$ & $0.0468(0.0124)$ & $0.0483(0.0140)$ & $0.0502(0.0124)$ & $0.0510(0.0119)$ \\
\hline \multirow{3}{*}{$(50,15)$} & $\lambda$ & $0.0510(0.0067)$ & $0.0546(0.0064)$ & $0.0512(0.0056)$ & $0.0492(0.0051)$ \\
\hline & $\theta_{1}$ & $0.0348(0.0042)$ & $0.0359(0.0075)$ & $0.0382(0.0076)$ & $0.0368(0.0062)$ \\
\hline & $\theta_{2}$ & $0.0459(0.0120)$ & $0.0468(0.0137)$ & $0.0500(0.0121)$ & $0.0500(0.0115)$ \\
\hline \multirow{3}{*}{$(50,20)$} & $\lambda$ & $0.0481(0.0065)$ & $0.0541(0.0062)$ & $0.0503(0.0053)$ & $0.0491(0.0050)$ \\
\hline & $\theta_{1}$ & $0.0330(0.0040)$ & $0.0335(0.0072)$ & $0.0368(0.0073)$ & $0.0369(0.0058)$ \\
\hline & $\theta_{2}$ & $0.0454(0.0116)$ & $0.0462(0.0134)$ & $0.0497(0.0118)$ & $0.0485(0.0113)$ \\
\hline
\end{tabular}

and Exchanges in Science and Technology Program of Shaanxi Province (2016KW-033).

\section{References}

[1] D. Han and D. Kundu, "Inference for a step-stress model with competing risks for failure from the generalized exponential distribution under type-I censoring," IEEE Transactions on Reliability, vol. 64, no. 1, pp. 31-43, 2015.

[2] S. Roy and C. Mukhopadhyay, "Maximum likelihood analysis of multi-stress accelerated life test data of series systems with competing log-normal causes of failure," Journal of Risk and Reliability, vol. 229, no. 2, pp. 119-130, 2015.

[3] X. P. Zhang, J. Z. Shang, X. Chen, C. H. Zhang, and Y. S. Wang, "Statistical inference of accelerated life testing with dependent competing failures based on copula theory," IEEE Transactions on Reliability, vol. 63, no. 3, pp. 764-780, 2014.

[4] Y.-M. Shi, L. Jin, C. Wei, and H.-B. Yue, "Constant-stress accelerated life test with competing risks under progressive type-II hybrid censoring," Advanced Materials Research, vol. 712-715, pp. 2080-2083, 2013.
[5] A. Xu and Y. Tang, "Objective Bayesian analysis of accelerated competing failure models under Type-I censoring," Computational Statistics and Data Analysis, vol. 55, no. 10, pp. 2830-2839, 2011.

[6] M. Wu, Y. Shi, and Y. Sun, "Inference for accelerated competing failure models from Weibull distribution under Type-I progressive hybrid censoring," Journal of Computational and Applied Mathematics, vol. 263, pp. 423-431, 2014.

[7] N. Balakrishnan and D. Han, "Exact inference for a simple stepstress model with competing risks for failure from exponential distribution under type-II censoring," Journal of Statistical Planning and Inference, vol. 138, no. 12, pp. 4172-4186, 2008.

[8] D. Han and N. Balakrishnan, "Inference for a simple step-stress model with competing risks for failure from the exponential distribution under time constraint," Computational Statistics and Data Analysis, vol. 54, no. 9, pp. 2066-2081, 2010.

[9] A. M. Abd-Elfattah, A. S. Hassan, and S. G. Nassr, "Estimation in step-stress partially accelerated life tests for the Burr type XII distribution using type I censoring," Statistical Methodology, vol. 5, no. 6, pp. 502-514, 2008.

[10] A. A. Ismail, "Bayes estimation of Gompertz distribution parameters and acceleration factor under partially accelerated 
life tests with type-I censoring," Journal of Statistical Computation and Simulation, vol. 80, no. 11, pp. 1253-1264, 2010.

[11] A. A. Ismail, "Estimating the parameters of Weibull distribution and the acceleration factor from hybrid partially accelerated life test," Applied Mathematical Modelling, vol. 36, no. 7, pp. 29202925, 2012.

[12] T. A. Abushal and A. A. Soliman, "Estimating the Pareto parameters under progressive censoring data for constantpartially accelerated life tests," Journal of Statistical Computation and Simulation, vol. 85, no. 5, pp. 917-934, 2015.

[13] A. A. Ismail and A. M. Sarhan, "Optimal design of step-stress life test with progressively type-II censored exponential data," International Mathematical Forum, vol. 4, no. 37-40, pp. 19631976, 2009.

[14] H. Jeffreys, The Theory of Probability, Oxford University Press, London, UK, 1998.

[15] Q. Guan, Y. Tang, and A. Xu, "Objective Bayesian analysis for bivariate Marshall-Olkin exponential distribution," Computational Statistics and Data Analysis, vol. 64, pp. 299-313, 2013.

[16] M. Han, "E-Bayesian estimation of failure probability and its application," Mathematical and Computer Modelling, vol. 45, no. 9-10, pp. 1272-1279, 2007.

[17] M. Han, "E-Bayesian estimation and hierarchical Bayesian estimation of failure probability," Communications in StatisticsTheory and Methods, vol. 40, no. 18, pp. 3303-3314, 2011.

[18] N. L. Johnson, S. Kotz, and N. Balakrishnan, Continuous Univariate Distributions, vol. 1, Wiley-Interscience, New York, NY, USA, 2nd edition, 1994.

[19] A. A. Soliman, "Estimations for Pareto model using general progressive censored data and asymmetric loss," Communications in Statistics. Theory and Methods, vol. 37, no. 8-10, pp. 1353-1370, 2008.

[20] H. T. Davis and M. L. Feldstein, "The generalized Pareto law as a model for progressively censored survival data," Biometrika, vol. 66, no. 2, pp. 299-306, 1979.

[21] C. M. Harris, "The Pareto distribution as a queue service discipline," Operations Research, vol. 16, no. 2, pp. 307-313, 1968.

[22] M. Bourguignon, H. Saulo, and R. N. Fernandez, "A new Paretotype distribution with applications in reliability and income data," Physica A: Statistical Mechanics and Its Applications, vol. 457, pp. 166-175, 2016.

[23] M. Doostparast, M. G. Akbari, and N. Balakrishnan, "Bayesian analysis for the two-parameter Pareto distribution based on record values and times," Journal of Statistical Computation and Simulation, vol. 81, no. 11, pp. 1393-1403, 2011.

[24] J. O. Berger, Statistical Decision Theory and Bayesian Analysis, Springer, New York, NY, USA, 2nd edition, 1985.

[25] D. N. P. Murthy, M. Xie, and R. Jiang, "Weibull models," in Wiley Series in Probability and Statistics, John Wiley and Sons, Hoboken, NJ, USA, 2004.

[26] W. B. Nelson, "Statistical methods for accelerated life test data the inverse power law model," General Electric Co. Tech. Rep 71-C011, Schenectady, New York, NY, USA, 1970.

[27] N. Balakrishnan and R. A. Sandhu, "A simple simulational algorithm for generating progressive Type-II censored samples," The American Statistician, vol. 49, no. 2, pp. 229-230, 1995. 


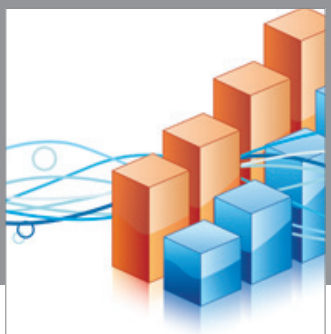

Advances in

Operations Research

vatem alat4

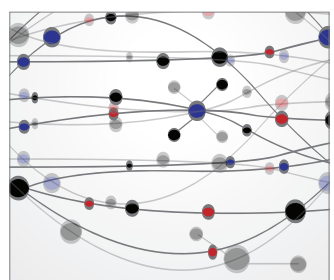

\section{The Scientific} World Journal
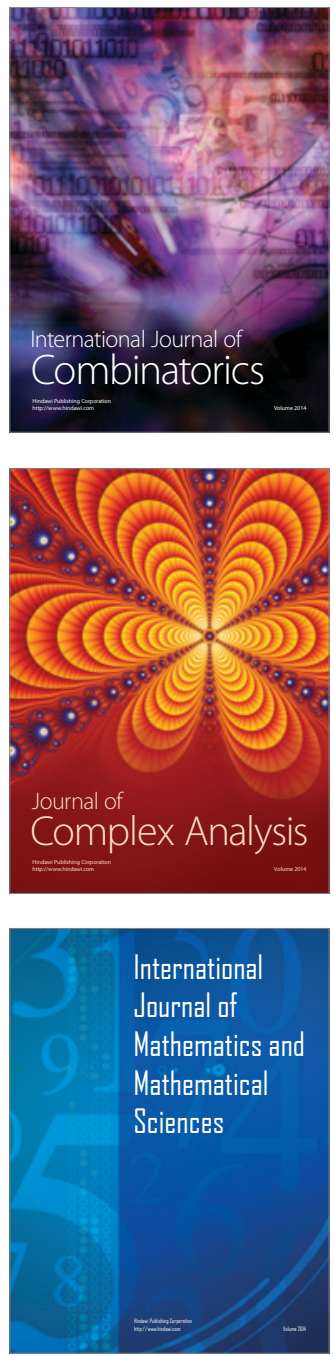
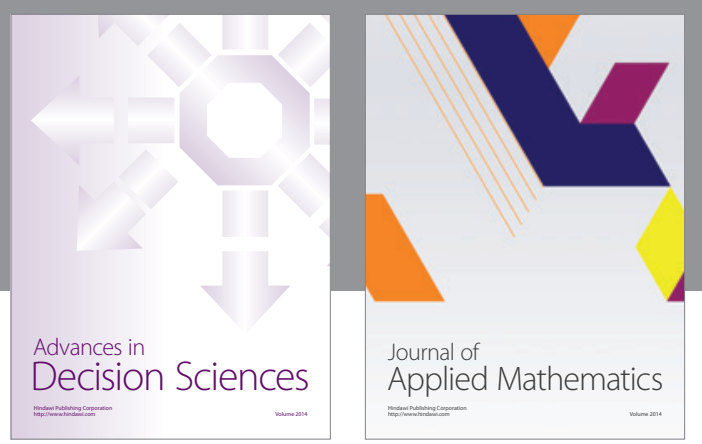

Algebra

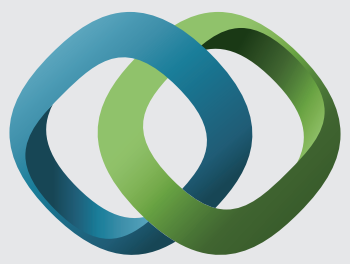

\section{Hindawi}

Submit your manuscripts at

http://www.hindawi.com
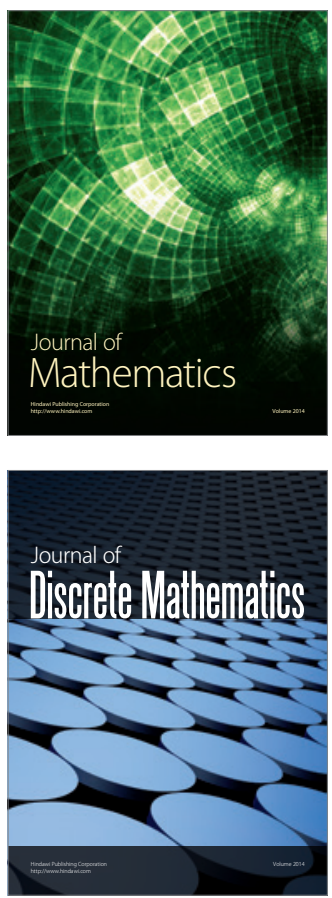

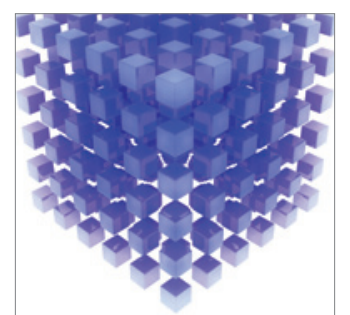

Mathematical Problems in Engineering
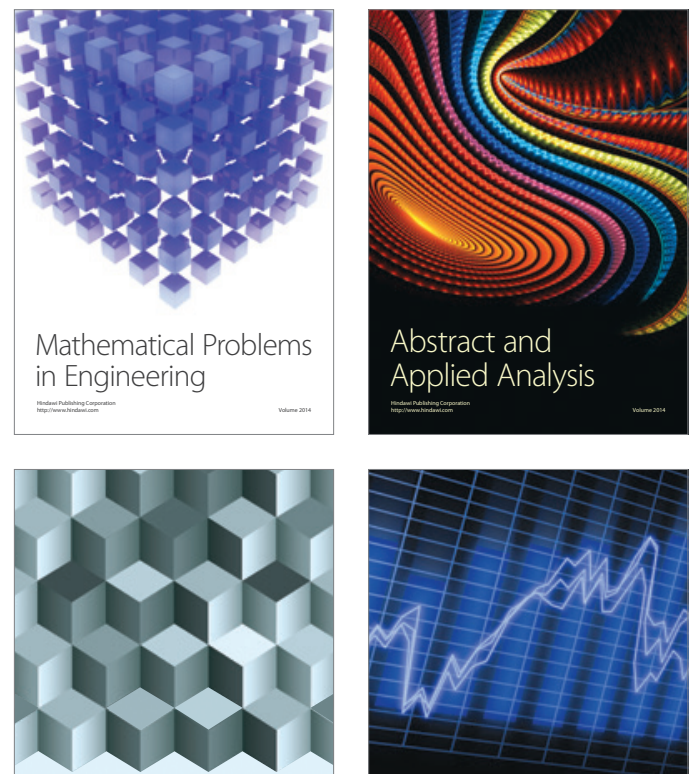

Journal of

Function Spaces

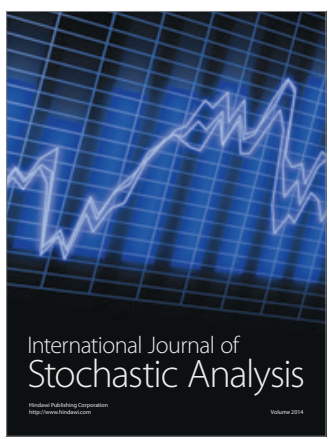

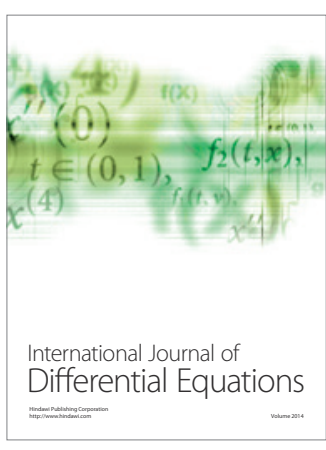
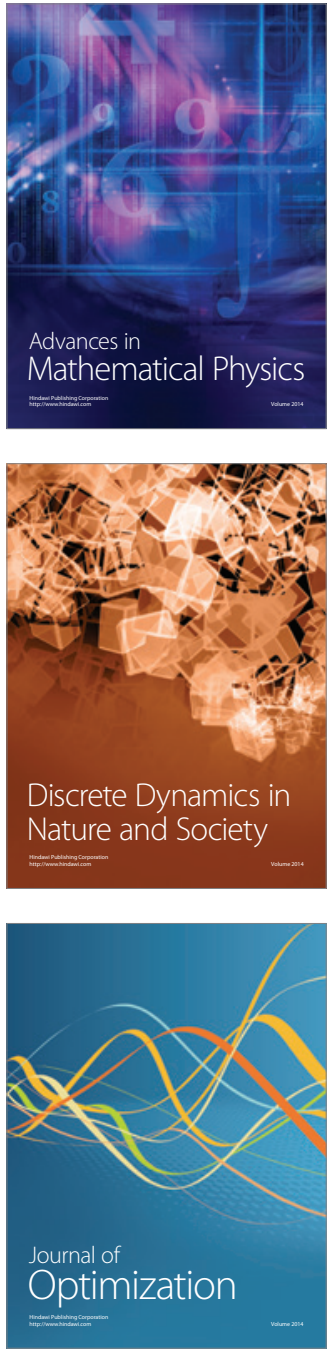